C-A/AP/\#123

November 2003

Beam Scrubbing for RHIC Polarized Proton Operation

S.Y. Zhang, W. Fischer, H. Huang, T. Roser

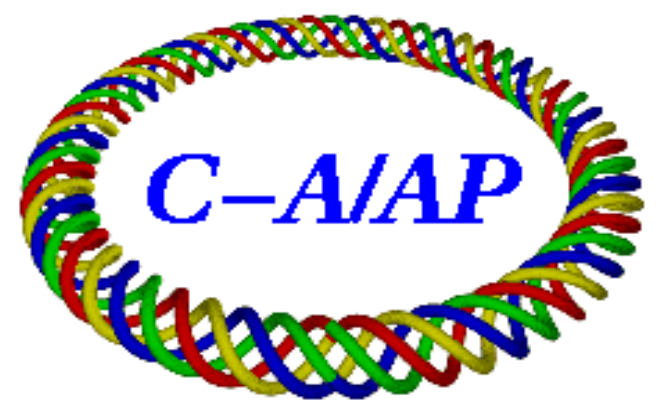

Collider-Accelerator Department

Brookhaven National Laboratory

Upton, NY 11973 


\title{
Beam Scrubbing for RHIC Polarized Proton Operation
}

\author{
S.Y. Zhang, W. Fischer, H. Huang, T. Roser
}

November 19, 2003

\begin{abstract}
One of the intensity limiting factor of RHIC polarized proton operation is the electron cloud induced pressure rise. A beam scrubbing study shows that with a reasonable period of time of running high intensity 112-bunch proton beam, the pressure rise can be reduced, allowing higher beam intensity.
\end{abstract}

\section{Introduction}

The basic beam scrubbing was discussed in [1], where it was shown that with the electron dose of $1 \mathrm{mC} / \mathrm{mm}^{2}$, the secondary electron yield can be reduced from 2.2 to 1.2 for stainless steel surface. The dose was specified for $500 \mathrm{eV}$ electrons. CERN SPS was the first one to implement the beam scrubbing for a rather long time of 10 days. At the end of the period, the LHC beam design intensity was achieved in the SPS for the first time [2]. LANL PSR observed beam scrubbing effect in a period of months of high intensity running [3]. Based on these results, a beam scrubbing by extending the beam store time was proposed for SNS [4].

The dose of $1 \mathrm{mC} / \mathrm{mm}^{2}$ is very large. Applying it in a time period of 24 hours with $500 \mathrm{eV}$ electrons, the pressure rise in an usual accelerator beam chamber will be higher than $5 \times 10^{-6}$ Torr [4], and vacuum pumps will stop functioning, followed by the valve closing to prevent damage of the devices.

The usual electron multipacting generated electrons have an energy distributed from very low, a few $\mathrm{eV}$, to around $300 \mathrm{eV}$. It is difficult to detect and calibrate these electrons with respect to the equivalent $500 \mathrm{eV}$ electrons' 
dose. On the other hand, the pressure rise, which is generated by the electron stimulated gas desorption, represents the scrubbing effect of the total electron dose. If the low energy electrons' dose is not contributing to pressure rise, it also not effective for beam scrubbing. In fact, electrons with the energy less than $20 \mathrm{eV}$ contribute very little to either pressure rise and beam scrubbing. Using beam induced pressure rise as a measure of beam scrubbing bypasses complications in using the electrons' dose. Therefore, one may consider to use pressure rise times hours as a practical dose measurement unit in beam scrubbing. Some cautions, however, are needed. Any pressure rise higher than $5 \times 10^{-6}$ Torr cannot be considered proper beam scrubbing, since the vacuum pumps will not work, and nonlinear pumping as a function of pressure level needs to be considered. The pumping capacity peaks at $10^{-7}$ Torr, and reduces to less than $20 \%$ at $5 \times 10^{-6}$ Torr [5]. This issue will be revisited later in this article.

For the RHIC, it was questioned if it is possible to get effective beam scrubbing within a tolerable time of period. The RHIC electron cloud induced pressure rise was very unevenly distributed in the warm sections. Beam scrubbing may be effective at the location(s) with the highest pressure rise, while not affecting many other locations. The scenario of scrubbing one location, followed by another newly emerged location, implies a longer scrubbing time than at other machines, such as SPS, and therefore, not practical for RHIC.

During the 2003 polarized proton run, a beam scrubbing study was performed. Actual beam scrubbing time was much less than the planned 2 hours. However, a non-trivial beam scrubbing effect was observed not only in the locations with highest pressure rise, Bo2 and Bi8, but also in most of the single beam straight sections. This not only confirmed that beam scrubbing is indeed a countermeasure to the electron cloud, but also showed the feasibility of applying beam scrubbing in RHIC proton beam operation to allow for higher beam intensities.

Two types of pressure rise affect the heavy ion operation in the RHIC [6]: the electron cloud induced pressure rise at the injection and the transition pressure rise. The latter currently poses a more serious limitation, preventing 112-bunch operations at high intensity. For proton running, however, electron cloud induced pressure rise is currently the only limit. Applying high intensity 112-bunch beam over a reasonable period time for beam scrubbing the pressure rise can be reduced, allowing high intensity beam operation.

In this note, the beam scrubbing study will be presented, followed by 

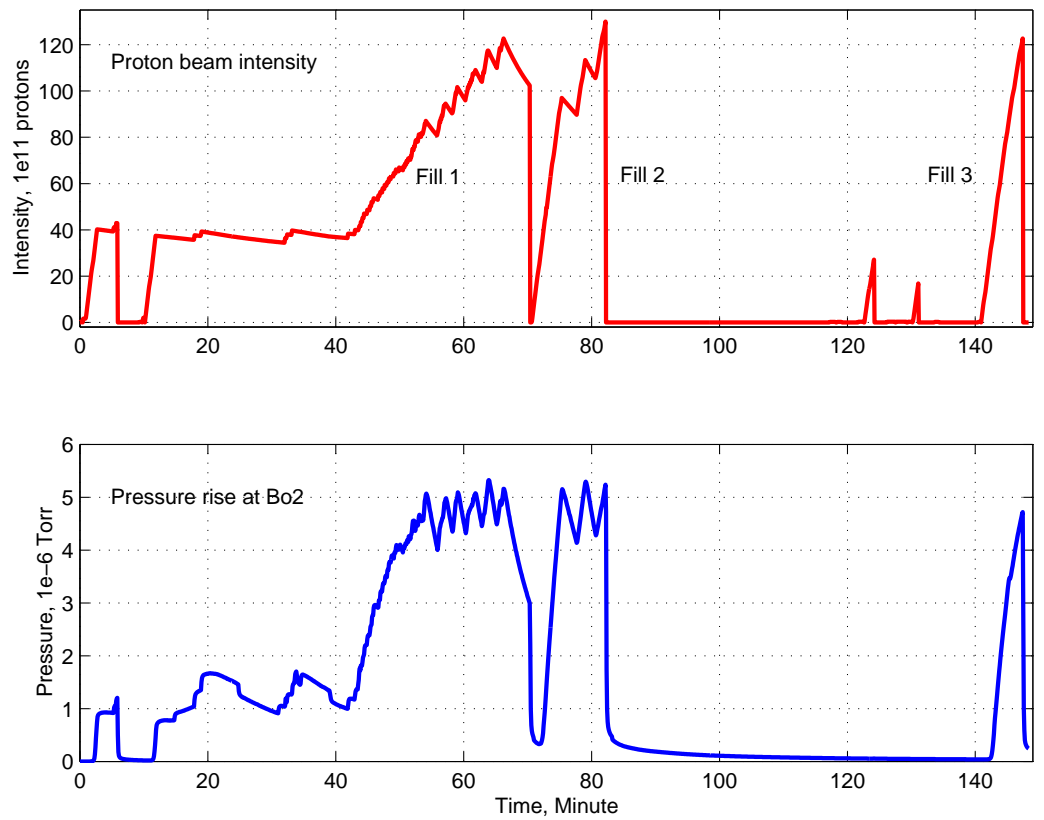

Figure 1: Beam scrubbing: Beam intensity and pressure rise at Bo2.

discussion.

\section{Beam scrubbing study}

The study was performed May 30, 2003, using the proton beam with the 112-bunch injection pattern. The proton bunch intensity was high, up to $2 \times 10^{11}$ per bunch. The beam was injected manually, observing the pressure rise closely to let it not exceed $5 \times 10^{-6}$ Torr. The highest pressure rise was at Bo2, i.e. blue ring Q3-Q4 straight section at 2'o clock.

As shown in Fig.1, when the beam intensity first reached $80 \times 10^{11}$ protons, the pressure rise at Bo2 reached $4.6 \times 10^{-6}$ Torr. With more beam injected, the pressure rise reached a little higher than $5 \times 10^{-6}$ Torr, then the injection was stopped. The pressure started dropping, partly because of the decreasing beam intensity, and maybe partly because of the short term beam scrubbing effect. Once the pressure dropped to about $4 \times 10^{-6}$ Torr, injection was resumed to get a high pressure rise again. After all 112 bunches were injected in the ring, the beam was dumped, and the next fill started. 
In the second fill, it required a total intensity of $90 \times 10^{11}$ protons to reach the same pressure rise of $4.6 \times 10^{-6}$ Torr. No only was the total intensity higher than when the first fill reached this pressure rise, but the average bunch intensity was $1.7 \times 10^{11}$ per bunch, much higher than for the first fill with $1.4 \times 10^{11}$ per bunch. The higher bunch intensity should be considered stronger electron multipacting driver.

The second fill was terminated by a loss monitor permit pull. The reason was not very clear, but one of the RF cavity tripped off at the same time. After about one hour, the third injection was attempted for possible ramping, but the beam was terminated again when 72 bunches were injected, possibly by yellow beam loss in the ring. It took the third injection a total beam intensity of $120 \times 10^{11}$ protons to reach the pressure rise of $4.6 \times 10^{-6}$ Torr at Bo2. The bunch intensity was as high as for the second fill, and the bunch length was shorter. For the first and second fills, the bunch length measured at FWHM (full width half maximum) was $7.3 \mathrm{ns,} \mathrm{whereas} \mathrm{for} \mathrm{the} \mathrm{third} \mathrm{fill}$ the first 48 bunches were 5 ns long, followed by the remaining 24 bunches filled with 7.3 ns bunch length.

In Table 1 the beam properties of the 3 fills are summarized when the pressure rise first reached $4.6 \times 10^{-6}$ Torr at Bo2. The electron multipacting driving capability getting stronger from fill 1 to 3 . Along with increasing beam intensities, the constant pressure rise at Bo2 supports the beam scrubbing effect.

\begin{tabular}{cccc} 
Fill & $\begin{array}{c}\text { Total intensity } \\
10^{11} \text { protons }\end{array}$ & $\begin{array}{c}\text { Ave. bunch intensity } \\
10^{11} \text { protons }\end{array}$ & $\begin{array}{c}\text { Ave. bunch length } \\
n s, \text { FWHM }\end{array}$ \\
\hline 1 & 80 & 1.4 & 7.3 \\
2 & 90 & 1.7 & 7.3 \\
3 & 120 & 1.7 & 6 \\
\hline
\end{tabular}

Table 1: Beam property when reaching $4.6 \times 10^{-6}$ Torr at Bo2

\section{Data analysis}

To study the beam scrubbing effect at different locations, all data from fast logged gauges in the warm sections of the ring (one sampling per second) were collected. These include 12 each at pw 3.2 of the Q3-Q4 straight sections, 


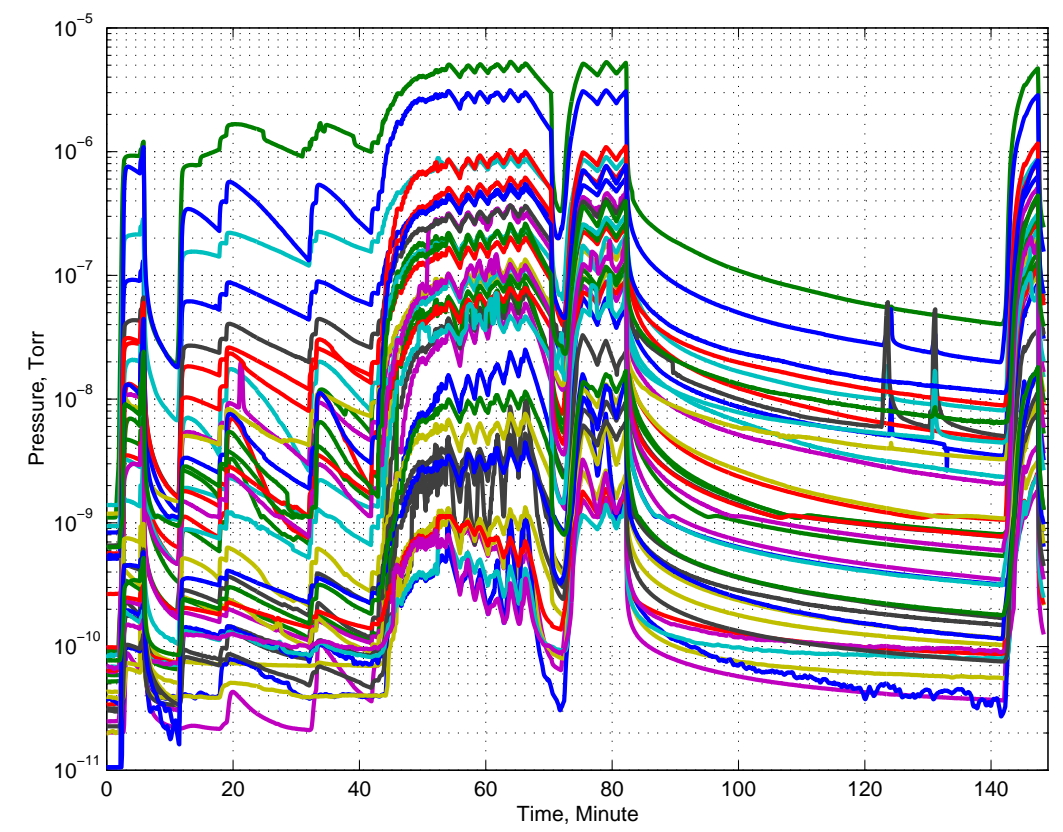

Figure 2: The pressure rises at all 37 fast logged gauges in RHIC warm sections during the beam scrubbing study. The pressure rise spans in 4 decades.

and 2 special ones at pw 3.1 at 10 o'clock and 4 o'clock, for abort kicker and RF cavities monitoring. Gauges labeled pw 3.2 are located at the center of the straight sections, and pw 3.1 is close to Q3. Also, there are 4 gauges for the 6 interaction regions, a total of 24 . One of these, at 8 o'clock, was not functioning. Pressure rises at a total of 37 locations, with the pressure rise of the first fill of $5 \times 10^{-6}$ Torr at Bo 2 and several at around $5 \times 10^{-10}$ Torr, are shown in Fig.2. The pressure rise spans 4 decades. Note that this is a 112-bunch injection pattern with the an intensity of up to $2 \times 10^{11}$ per bunch. Almost all location have shown some pressure rise. Electron signals obtained by several electron detectors at the locations with high pressure rise had shown close relation with the pressure rise, and signals have shown the typical pattern of electron multipacting [7]. Electron signals for very low pressure rise, $10^{-9}$ Torr or below, cannot be detected, and the mechanism there is less clear.

4 groups of locations are identified with similar pressure rise behavior. The first group consists of 2 locations with the highest pressure rise of $3 \times$ 

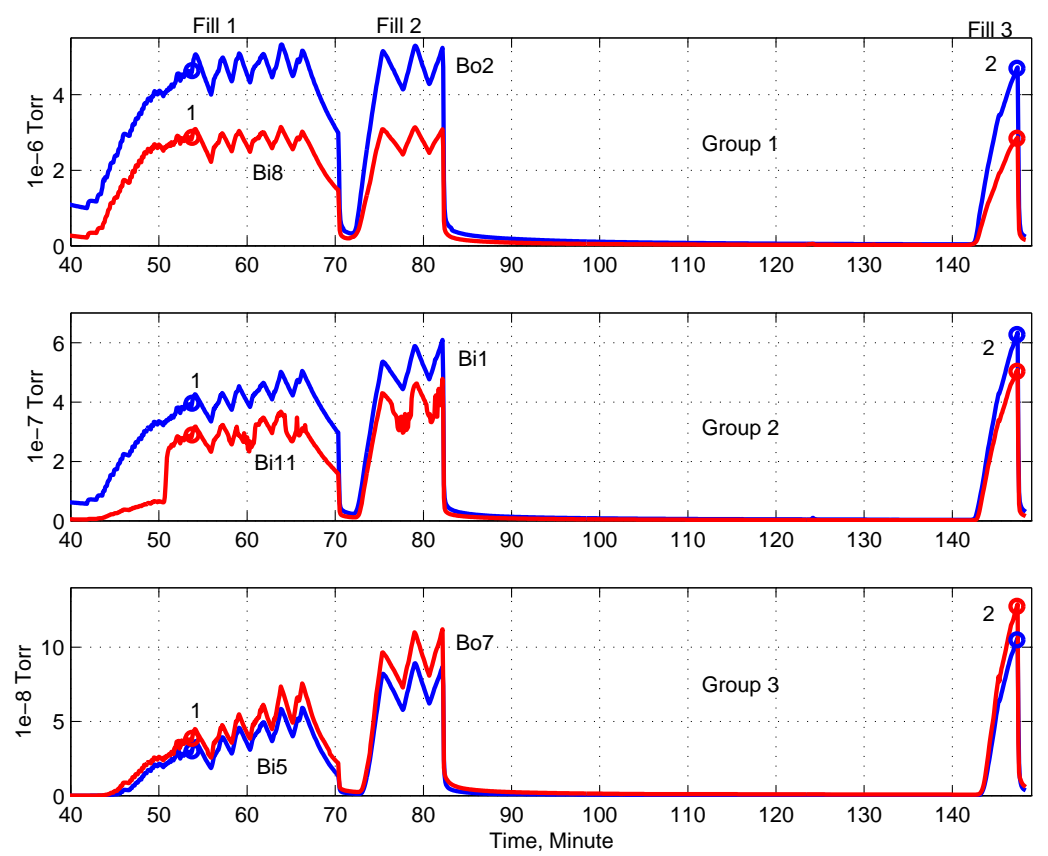

Figure 3: Typical pressure rise pattern for three different groups, with the high, medium, and low pressure rises.

$10^{-6}$ Torr to $5 \times 10^{-6}$ Torr. The second group consists of about 10 locations with a pressure rise of $10^{-7}$ Torr to $10^{-6}$ Torr. The third group consists of more than 10 locations with the pressure rise of $10^{-8}$ Torr to $10^{-7}$ Torr. Below a pressure rise of $10^{-8}$ Torr, the data are probably not reliable regarding to beam scrubbing, which is the fourth group.

With much stronger beam in the Fill 3, the first group showed the beam scrubbing effect, i.e., the pressure rise was not higher than that for Fill 1. The second group showed less scrubbing effect, while for the third group the effect is probably negligible.

Typical cases for the first 3 groups are shown in Fig.3. Pressure rises at Bo2 and Bi8 are shown for the first group. Pressure rises at Bi1 and Bi12 are shown for the group 2. Note that the solenoids were on at Bi12 for the first 50 minutes, which limited the pressure rise there. Later, there was also solenoid study there causing wiggles in the pressure rise. Finally, pressure rises at $\mathrm{Bo} 7$ and $\mathrm{Bi} 5$ are shown for the third group.

The pressure rises at Bo7 and Bi5 peaked at the point 2 in Fig.3, for Fill 
3, is about 2.5 times higher than that at the point 1, for Fill 1. These pressure rises are approximately proportional to the beam strength of electron multipacting driving, with negligible scrubbing effect. This is compared with the group 1, where the flat pressure rises at points 1 and 2 shows the beam scrubbing effect.

To compare the beam scrubbing effect at all 37 locations,

- The integral of the pressure rise starting from the beginning of the study to the end of Fill 2 is used as the 'dose', in units of $10^{-6}$ Torr $\times$ hour.

- The scrubbing effect is represented by the ratio of this 'dose' divided by the peak pressure rise at the Fill 3 averaged in 1 minute.

- This scrubbing effect is normalized with the pressure rise at Bi5, which is assumed to have negligible scrubbing effect. Therefore, the scrubbing effect is 1 for Bi5.

In Fig.4, the beam scrubbing effect for all warm sections in the ring is shown. The 12 red dots are the Q3-Q4 single beam straight sections. The two black dots are for sections 10 and 4, monitoring for the abort kicker and $\mathrm{RF}$ cavities. The 23 blue dots are at the 6 interaction regions.

Observations of the data shown in Fig.4 are as follows:

- The two red dots at the 'dose' of 0.02 to $0.0310^{-6}$ Torr $\times$ hour show scrubbing effect at Bo7 and Bi5. As shown in Fig.3, at these two locations there is negligible scrubbing effect.

- Below the 'dose' level of $0.0210^{-6}$ Torr $\times$ hour, the scattered distribution of the scrubbing effect around 1 shows that to choose $\operatorname{Bi} 5$ to represent the negligible scrubbing effect is appropriate.

- Above this 'dose' level, the scrubbing effect increases with the larger 'dose'. The largest scrubbing effect is about 2.5 at Bo2, which is consistent with the pressure rises for 3 fills shown in Fig.3.

The scrubbing effect shown in Fig.4 is not linearly proportional to the 'dose' derived from the pressure rise. An important issue here is the nonlinear pumping speed. As mentioned previously, the peak pumping capacity is at a pressure of $10^{-7}$ Torr. At $5 \times 10^{-6}$ Torr, it is reduced to less than $20 \%$. 


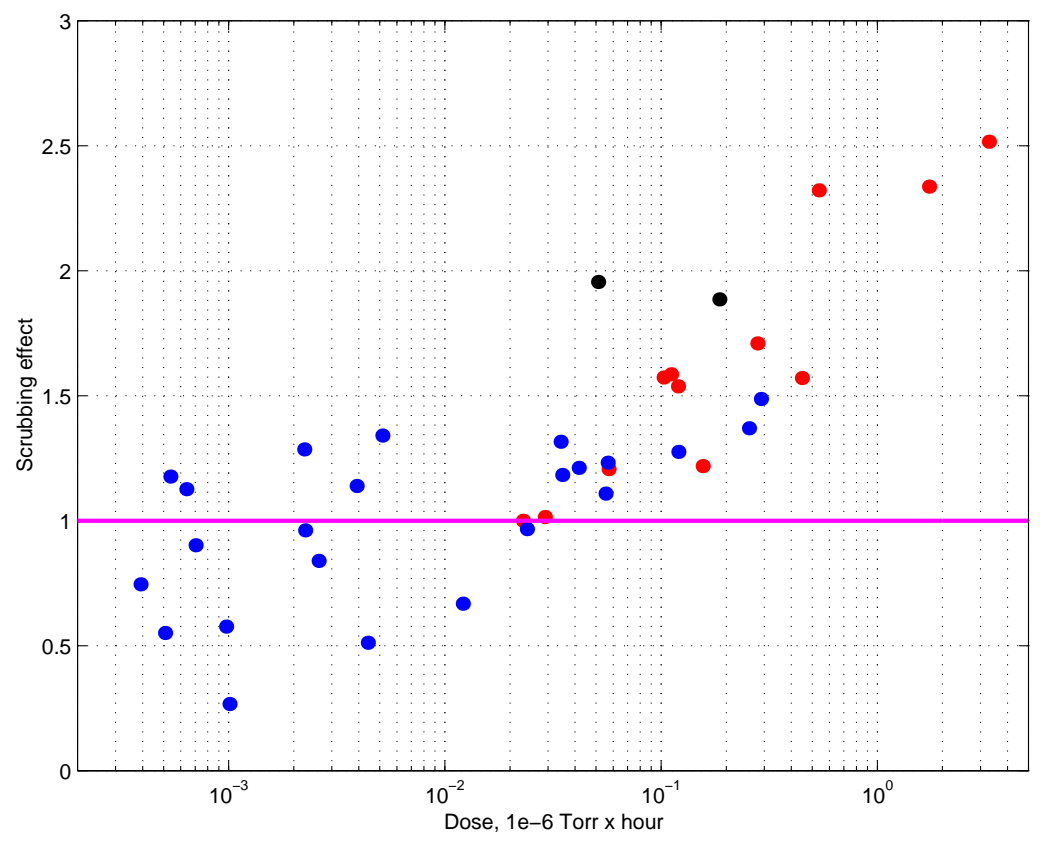

Figure 4: Beam scrubbing effect. Unit 1 means no scrubbing effect. Red dots are for the Q3-Q4 single beam straight sections. Black dots are special ones in sections 4 and 10 . Blue dots are for interaction regions.

Therefore, Fig.4 should not be used to predict the scrubbing effect for other time scales.

SPS beam scrubbing has shown that with the pressure rise of about $5 \times$ $10^{-6}$ Torr for 24 hours, the pressure rise with the initial beam was reduced by a factor of 100 . This 'dose' is $12010^{-6}$ Torr $\times$ hour, and is not inconsistent with the result of this study: the 'dose' of $3.210^{-6}$ Torr $\times$ hour received at Bo2 resulted in a factor of 2.5 pressure rise reduction.

Assuming that the RHIC scrubbing takes 4 hours then the total 'dose' will be $2010^{-6}$ Torr $\times$ hour, and the pressure rise would be reduced in a factor larger than 15. This may allow injection of 112-bunch proton beam with a bunch intensity of $1 \times 10^{11}$ protons, ready for acceleration. Since in the RHIC beam induced pressure rise only occurs in warm sections, rather than the whole ring, beam instability is not of much concern so far, and limited pressure rise reduction might be sufficient to allow high intensity beam injection. It is also known that, once the beam is accelerated, the pressure will reduce, and pressure rise is not a problem at the store. In 
comparison, at the SPS, it is required to eliminate the electron cloud [8], which requires much more scrubbing.

\section{Discussion}

A brief summary and discussions follow:

- The beam scrubbing study at the RHIC has shown consistent results of the scrubbing effect at locations having pressure rises higher than $10^{-7}$ Torr. The higher the pressure rise, the better the scrubbing effect. The scrubbing equivalent of 40 minutes with a beam induced pressure rise of $5 \times 10^{-6}$ Torr has reduced the pressure rise by a factor of 2.5 , for the initial beam.

- Since the electron cloud induced pressure rise is the only limit in RHIC polarized proton high intensity operation, the beam scrubbing can be used to remove this limit, allowing 112-bunch high intensity operation.

- A total beam intensity of more than $120 \times 10^{11}$ protons, with an average bunch intensity of $1.7 \times 10^{11}$, induced pressure rise of less than $5 \times$ $10^{-6}$ Torr at the worst location at the end of the beam scrubbing study. This by itself has demonstrated the feasibility of using beam scrubbing to enable a 112-bunch, high intensity proton run in near future.

- A 4 hours of beam scrubbing, using both beams, may help to confirm the scrubbing effect. This could be done as part of the beam commissioning.

- In the same day of the beam scrubbing study, about 30 of the BPM electroncis modules in the tunnel failed [9]. For the high beam intensity and poor beam lifetime during the scrubbing study, the beam losses that exceeded significantly the beam losses in normal operation could have caused these damages. If true, then this will prevent scrubbing until all BPM electronics is moved into the alcoves, probably by the beginning of Run 5. A beam scrubbing test may take place at the end of Run 4, with the consideration of possible BPM repair costs. 


\section{Acknowledgment}

We would like to thank P. He, H.C. Hseuh, U. Iriso, R. Lee, F. Pilat, L. Smart, and D. Trbojevic for discussion and support.

\section{References}

[1] N. Hilleret et al, p. 2629, PAC, New York, 1999.

[2] J.M. Jimenez, Mini-Workshop on SPS Scrubbing run, CERN, 2002.

[3] R. Macek, ECLOUD'02, CERN, April, 2002.

[4] S.Y. Zhang et al, Beam Scrubbing Strategy for Electron-cloud Suppression in the Spallation Neutron Source Ring, PAC, 2003.

[5] U. Iriso et al. in preparation.

[6] S.Y. Zhang et al, RHIC Pressure Rise and Electron Cloud, PAC, 2003.

[7] H.C. Hseuh, private communication.

[8] F. Zimmermann, private communication.

[9] T. Satogata and P. Cerniglia, private communication. 